\title{
含硫香精香料的合成进展
}

\author{
王明 $a$ 王翠红 ${ }^{*, a}$ 姜雪峰*,a,b \\ ( ${ }^{a}$ 华东师范大学化学与分子工程学院 上海市绿色化学与化工过程绿色化重点实验室 上海 200062) \\ ( ${ }^{b}$ 南开大学 元素有机化学国家重点实验室 天津 300071)
}

\begin{abstract}
摘要 随着科技水平的提高, 高精度的分析方法及设备被开发, 科学家也在食品中发现了越来越多的含硫化合物. 2015 年, 美国食品香料与萃取制造者协会公布的 2816 种被认为安全的香料中，含硫化合物有 388 多种，占总数的 $13 \%$ 以上. 含硫香料的主要表现为食物特别是副食和菜肴相关的香味, 其主要被用作食品香精及花香和果香型日用香精. 由于需求量的增加, 含硫香料的生产规模也在不断的增加, 其合成方法的研究也在不断的深入, 绿色、高效的构建方法 也不断的被报道. 对硫醚、硫醇、硫酯及多硫等香精香料分子的最新构建方法进行概述.
\end{abstract}

关键词＼cjkstart硫醚; 硫醇; 硫酯; 多硫; 香精; 香料

\section{Recent Progress in the Sulfur-Containing Perfume \& Flavors Construction}

\author{
Wang, Ming ${ }^{a}$ Wang, Cuihong ${ }^{*, a} \quad$ Jiang, Xuefeng ${ }^{*, a, b}$ \\ ( ${ }^{a}$ Shanghai Key Laboratory of Green Chemistry and Chemical Process, School of Chemistry and Molecular Engineering, \\ East China Normal University, Shanghai 200062) \\ ( ${ }^{b}$ State Key Laboratory of Elemento-organic Chemistry, Nankai University, Tianjin 300071)
}

\begin{abstract}
Scientists have found more and more sulfur compounds in food with the development of science and technology, high-precision analytical methods and equipment. In 2015, Flavor and Extract Manufactures Association of the United State published 2816 safe flavors, and there are more than 388 sulfur-containing compounds, accounting for more than $13 \%$. The main smell of sulfur-containing flavors is food, especially the non-staple food and dishes. It is mainly used as food flavor, flower flavor and fruity flavor. Due to the increase of sulfur-containing perfume and flavors demand, its production scale is increasing, the research on synthesis methods is also deepening, and the green and efficient construction methods are also constantly reported. In this review, the recent progress in the construction of sulfide, thiol, thioester and polysulfide perfume \& flavors is summarized.
\end{abstract}

Keywords sulfide; thiol; thioester; polysulfide; perfume; flavors

有机含硫化合物由于具有独特的气味性质，在很多 领域具有不可替代的用途. 例如, 1937 年 3 月 18 日, 美 国德克萨斯州的新伦敦学校发生了巨大的天然气爆炸 事件, 造成近三百名师生死亡, 从此以后, 美国德克萨 斯州立法强制要求在无味的天然气中添加有臭味的甲 硫醇或乙硫醇, 能让人们在天然气泄露的第一时间闻到 气味并察觉危险. 这种做法迅速被传开, 慢慢成为全球 的统一规范. 含硫化合物纯品强烈的令人不愉悦的气味
很难将其和香料联系起来, 但随着进一步的研究发现, 含硫化合物在低浓度的香味效果和高浓度时有着巨大 的差别. 例如低浓度的二甲硫醚蒸气会让人食欲减退, 高浓度的二甲硫醚蒸气则会对中枢神经系统有麻痹的 作用 ${ }^{[1]}$, 但二甲硫醚在 $0.5 \%$ 浓度时具有奶油、扇贝、浆 果等香味, 在 $1 \mathrm{mg} / \mathrm{kg}$ 时具有西红柿、玉米、奶制品香 味和淡淡的薄荷香，可用于草莓、玉米、奶油、鸡蛋、 焙烤食物等食品香精 ${ }^{[2]}$. 研究也发现, 即使含硫化合物

\footnotetext{
* Corresponding authors. E-mail: xfjiang@chem.ecnu.edu.cn; chwang@chem.ecnu.edu.cn Received March 29, 2019; revised April 22, 2019; published online May 10, 2019. Dedicated to the 100th anniversary of the birth of Professor Ruyu Chen. Project supported by the National Key Research and Development Program of China (No. 2017YFD0200500), and the National Natural Science Foundation of China (Nos. 21722202, 21672069, 21871089).

国家重点研发计划(2017YFD0200500)、国家自然科学基金(Nos. 21722202, 21672069, 21871089)资助项目.
} 
在食物中的含量用常规的分析方法难以检测得到, 但是 却在食物的香味中起主导作用, 对食物的风味有很大的 改变. 随着科技水平的提高, 高精度的分析方法及设备 被开发, 科学家在食品中发现了越来越多的含硫化合 物. 2015 年, 美国食品香料与萃取制造者协会(FEMA, Flavor and Extract Manufactures Association of the United State)公布的 2816 种被认为安全的香料中, 含硫化合物 有 388 多种, 占总数的 $13 \%$ 以上 ${ }^{[3]}$, 并且迄今为止, 含硫 香料的品种数量还在不断的增加.

含硫香料的主要表现为食物特别是副食和菜有相 关的香味, 如各种肉香、海鲜、洋葱、韭菜等香味特征, 其主要被用作食品香精及花香和果香型日用香精. 由于 需求量的增加, 含硫香料的生产规模也在不断的增加, 其合成方法的研究也在不断深入. 绿色、高效的构建方 法也不断被报道. 孙宝国 ${ }^{[4]}$ 在 2007 年《含硫香料化学》 一书中, 对含硫类香料分子已有完整总结, 本文在此基 础上, 对硫醚、硫醇、硫酯及多硫类香精香料分子近年 来发展的最新构建方法进行概述.

\section{1 硫醚类}

硫醚类化合物作为含硫香料中重要的一部分, 由于 其化学稳定性比硫醇高, 保持香味的质量较高, 已经得 到广泛的应用(表 1). 随着科学家对有机硫醚化合物认
识的深入，越来越多的硫梄类香料被人们所接受. 如最 简单的二甲基硫醚就具有洋苟心、苟心蒜、奶油、扇贝等 香味 ${ }^{[2,5]}$. 正是由于硫醚类香料实用性, 近年来化学工作 者发展了一系列高效构建硫醚的方法.

$\beta$-羰基硫醚类香精香料分子早前都是通过硫醇与 $\beta$ 羰基卤化物的亲核取代反应来构建 $(\text { Scheme } 1 \mathrm{a})^{[6]}$. 随后， 研究者发展了硫醇对环氧丙烷的开环得到 $\beta$-羟基硫醚, 再将其氧化为 $\beta$-羰基硫醚(Scheme $1 \mathrm{~b})^{[7]}$. 除此之外, 酮 化合物 6 制备成烯醇硅醚 7, 再将其氧化为 $\alpha$-差基酮 $\mathbf{8}$, 甲磺酰基化之后，接受硫醇化合物 9 的进攻，也可以构 建 $\beta$-羰基硫醚类香料分子 $\mathbf{3 b}$ (Scheme 1c) ${ }^{[8]}$. 1,3-二羰基 化合物 10 在碱条件下，与过硫或者硫醇也可以构建 $\beta$ 羰基硫醚类化合物 $\mathbf{1 1}$ (Scheme 1d) ${ }^{[9]}$. 同时，酮化合物 12 可以在碘、三苯基膦介导下，水溶剂中与亚磺酸钠盐 13 来构建 $\beta$-羰基硫醚类化合物 14 (Scheme 1e $)^{[10]}$.

$\gamma$-羰基硫醚的构建基本都是通过硫醇对 $\alpha, \beta$-不饱和 酮的 Michael 加成来构建. 例如香料分子 17 的合成就是 由呋喃甲硫醇 15 与 $\alpha, \beta$-不饱和酮 16 加成得到(Eq. 1) ${ }^{[11]}$.

$\beta$-羟基硫醚类香精香料分子的合成与 $\beta$-羰基硫醚类 香精香料分子类似，主要有两种方法. 一种方法例如香 料分子 19 的合成, 是由相应的硫醇对环氧丙烷 18 开环 得到 $\beta$-羟基硫醚(Scheme $2 \mathrm{a}$ ). 另一种合成方法例如香料 分子 22 的合成, 是由硫醇与 $\beta$-羰基卤化物 20 的亲核

表 1 硫醚类香精香料分子特征结构和香味

Table 1 Structure of thiol-containing flavors and their smell

结构类别




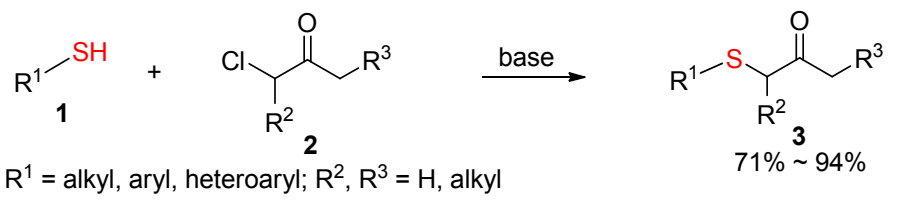<smiles>[R]CC(=O)CS[R][H]</smiles>

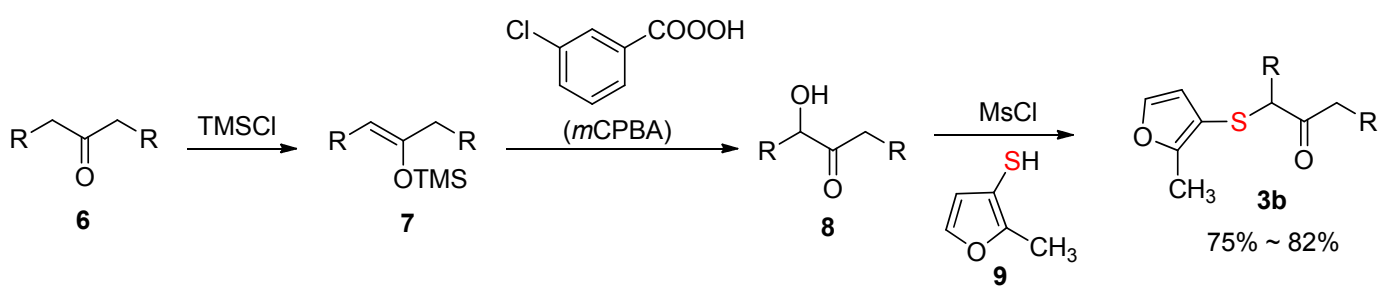

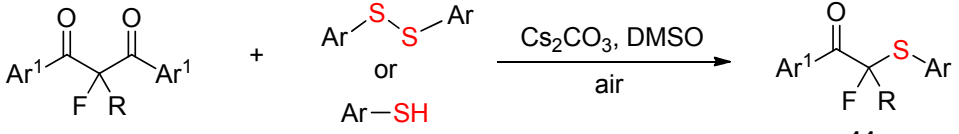

$$
\begin{aligned}
& \mathrm{R}=\mathrm{H} \text { or } \mathrm{F} \\
& 11 \\
& 44 \% \sim 98 \%
\end{aligned}
$$

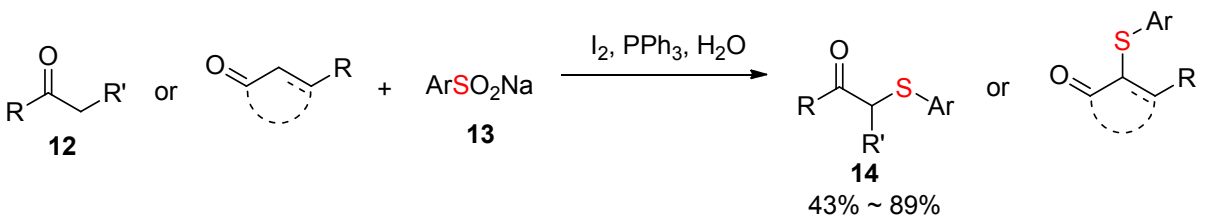

图式 $1 \beta$-羰基硫醚的构建

Scheme 1 Construction of $\beta$-carbonyl sulfide

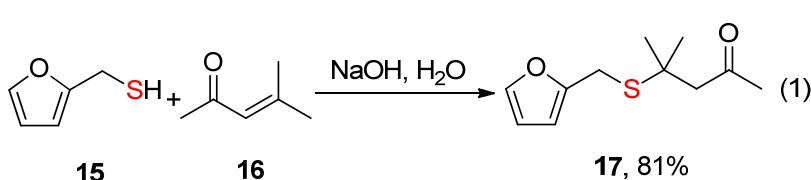

15

16

$17,81 \%$

取代, 再还原来构建 $(\text { Scheme 2b) })^{[12,7 b]}$.

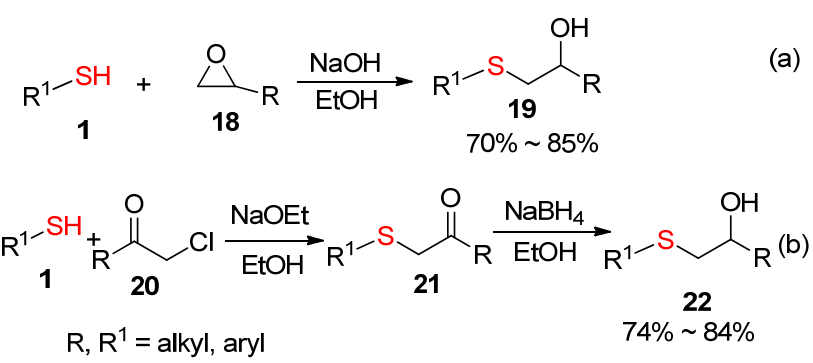

图式 $2 \beta$-差基硫醚的构建

Scheme 2 Construction of $\beta$-hydroxyl sulfide

$\gamma$-羟基硫醚俗称菠萝醇，天然存在于苹果、番茄、 啤酒、葡萄酒中. GB/T20824-2007 中将3-甲硫基丙醇规 定为芝麻香型白酒的特征风味物质, 并限定高度数酒中 3-甲硫基丙醇质量浓度须大于 $0.5 \mathrm{mg} / \mathrm{L}$, 低度数酒质量 浓度须大于 $0.4 \mathrm{mg} / \mathrm{L}^{[13]}(\mathrm{GB} / \mathrm{T}$ 20824-2007 芝麻香型白
酒, 中国标准出版社, 北京, 2007). 主要合成方法是由 相应的硫醇与 $\gamma$-着基烷基卤化物 23 通过亲核取代反应 合成得到(Scheme 3a) ${ }^{[14]}$. 也可以由相应的硫醇对 $\alpha, \beta$-不 饱和醛的 Michael 加成来合成(Scheme $3 b)^{[15]}$.

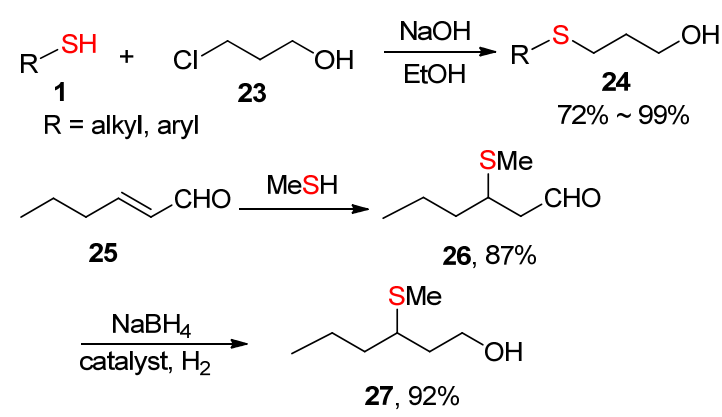

图式 $3 \gamma$-羟基硫醚的构建

Scheme 3 Construction of $\gamma$-hydroxyl sulfide

呋喃硫醚类化合物在香精香料分化合物中具有广 泛的用途，它的合成通常有以下几种方法. 一种方法如 香料分子 31 的合成，是由糠醛为原料，首先制备成相应 的缩醛 29, 再与相应的过硫 30 在还原条件下构建呋喃 硫醚分子 31 (Scheme 4a) ${ }^{[16]}$. 另外一种方法是通过呋喃 
-2-甲基过硫化合物 32 在还原条件下与烷基卤化物偶联 而得 $(\text { Scheme } 4 b)^{[17]}$. 呋喃硫醚类化合物还可以通过可 以通过呋喃和碘代乙酸乙酯反应并还原得到相应的呋 喃-2-乙基醇 36, 再经过磺酰化、硫代乙酰化、还原得到 呋喃-2-乙基硫醇 37, 最后呋喃-2-乙基硫醇烷基化得到 相应的呋喃硫醚 38 (Scheme $4 c)^{[18]}$.

1,4-二噻烷类化合物 41 是巯基醛(酤)的二聚体, 通 常表现出烤肉、牛肉和土豆香味等特征. 它可以由 $\alpha$-氯 代的醛(酮)39 与硫氢化钠先制备成 $\alpha$-颈基醛(酮)40, 再 二聚得到 1,4-二噻烷结构(Eq. 2) ${ }^{[19]}$.

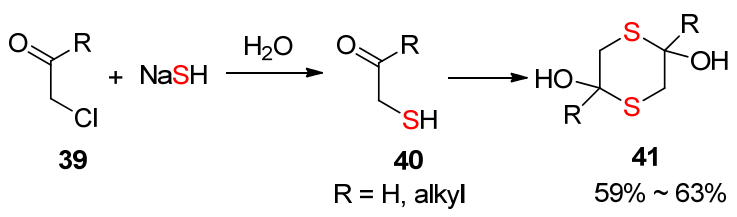

\section{2 硫醇类}

硫醇类分子是含硫香精香料分子中最重要的一类 之一，同时也是合成很多其它含硫香精香料分子的基本 原料. 尽管硫醇被人们认为具有恶臭的气味, 但是低浓 度的硫醇却能表现出食品香味. 例如, 常见的咖啡、香 油、葱蒜、烤肉等香味都是可以由硫醇类香料分子产生. 常见的硫醇类香精香料分子根据其结构特点可以分为 表 2 所示的七类骨架.

首先, 全碳硫醇类香料分子的合成可以由醇类化合 物直接硫化得到. 例如香料分子 43 的合成就是由相应 的三级醇 42 在 Lawesson 试剂条件下硫化得到(Scheme $5 a)^{[20]}$. 除了 Lawesson 试剂可以作为硫化试剂之外, 硫 脲作为无臭硫源, 也可以将醇类化合物硫化制备硫醇. 例如，醇 44 与硫脲经过中间体 46 可以制备得到香料分
子 47 (Scheme 5b), 化合物 47 表现出极低的阀值(0.014 $n g / L)$, 而且具有独特的桃香味，可用于香水制品和相应 的食品香精香料 ${ }^{[20]}$.

呋喃/四氢呋喃/二氢呋喃硫醇类骨架具有独特的烤 肉香味和咖啡香味. 尤其是 2-甲基-3-呋喃硫醇, 被认为 是目前为止最重要的肉味香料. 目前研究发现的肉香味 成分中关键组分均是 2-甲基-3-呋喃硫醇及其衍生物 ${ }^{[4]}$. 呋喃/四氢呋喃/二氢呋喃硫醇类化合物的合成除了传统 的环化反应之外，主要是通过硫醇对双键的加成以及醇 的硫化来实现. 例如四氢呋喃硫醇类香料分子 $\mathbf{5 0}$ 是由 硫代乙酸与二氢呋喃加成得到 49, 再水解即可合成四 氢呋喃硫醇化合物 50 (Scheme 6a) ${ }^{[21]}$. 也可以将呋喃-2醇类化合物 51 通过硫艮硫化得到呋喃-2-硫醇类化合物 52 (Scheme 6b) ${ }^{[22]}$.

$\beta$-羟基硫醇化合物是一类表现为果香味的香精香 料分子. 该类化合物的合成通常用不同硫试剂对环氧丙 烷衍生物的加成得到. 如 3-颈基-2-丁醇化合物 55 可以 由 2,3-二甲基环氧丙烷 53 和三甲基硅基保护的硫醚 54 合成得到(Eq. 3) ${ }^{[23]}$. 当用硫化氢代替硫化试剂 54 时，也 可以用来构建该类 $\beta$-差欮基硫醇化合物 ${ }^{[24]}$

$\gamma$-羟基硫醇类化合物主要表现出韭菜香味、洋葱香 味等刺激性香味，一些长链的 $\gamma$-羟基硫醇也表现出青 香 ${ }^{[25]}$. 该类化合物 59 的合成可以从烯丙醇 56 出发, 氧 化成 $\alpha, \beta$-不饱和醛 57 之后, 硫代乙酸对其 Michael 加成 得到 58, 再还原并脱去乙酰基保护即合成 $\gamma$-羟基硫醇化 合物 59 (Scheme 7) ${ }^{[26]}$.

$\beta$-羰基硫醇类化合物也是肉香味香料的重要来源. 该类化合物的合成如 Scheme 8 所示, $\alpha$-差基酮 60 的差基 用甲磺酰基保护得到化合物 61 之后，硫代乙酸钾亲核

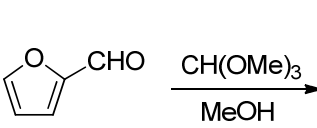

28

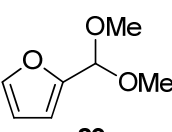

29

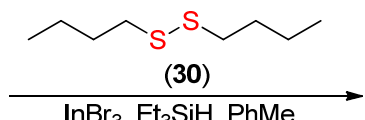

$\operatorname{lnBr}_{3}, \mathrm{Et}_{3} \mathrm{SiH}, \mathrm{PhMe}$

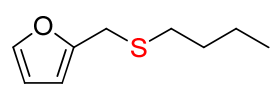

$31,34 \%$<smiles>c1coc(CSSCc2ccco2)c1</smiles><smiles>CCCCCBr</smiles>
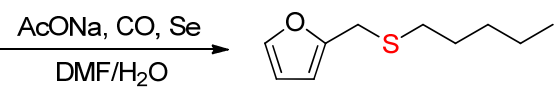

$34,90 \%$

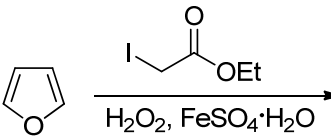

DMSO

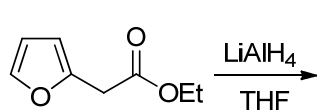

$35,92 \%$

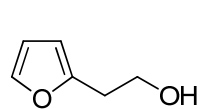

36, $64 \%$

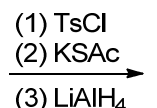

(3) $\mathrm{LiAlH}_{4}$<smiles>[R]C(I)[13CH2][13CH3]</smiles>

$37,90 \%$

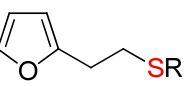

38 $32 \% \sim 87 \%$

图式 4 呋喃硫醚的构建

Scheme 4 Construction of furanyl sulfide 
表 2 硫醇类香精香料分子特征结构和香味

Table 2 The structure of thiol-containing flavors and their smell

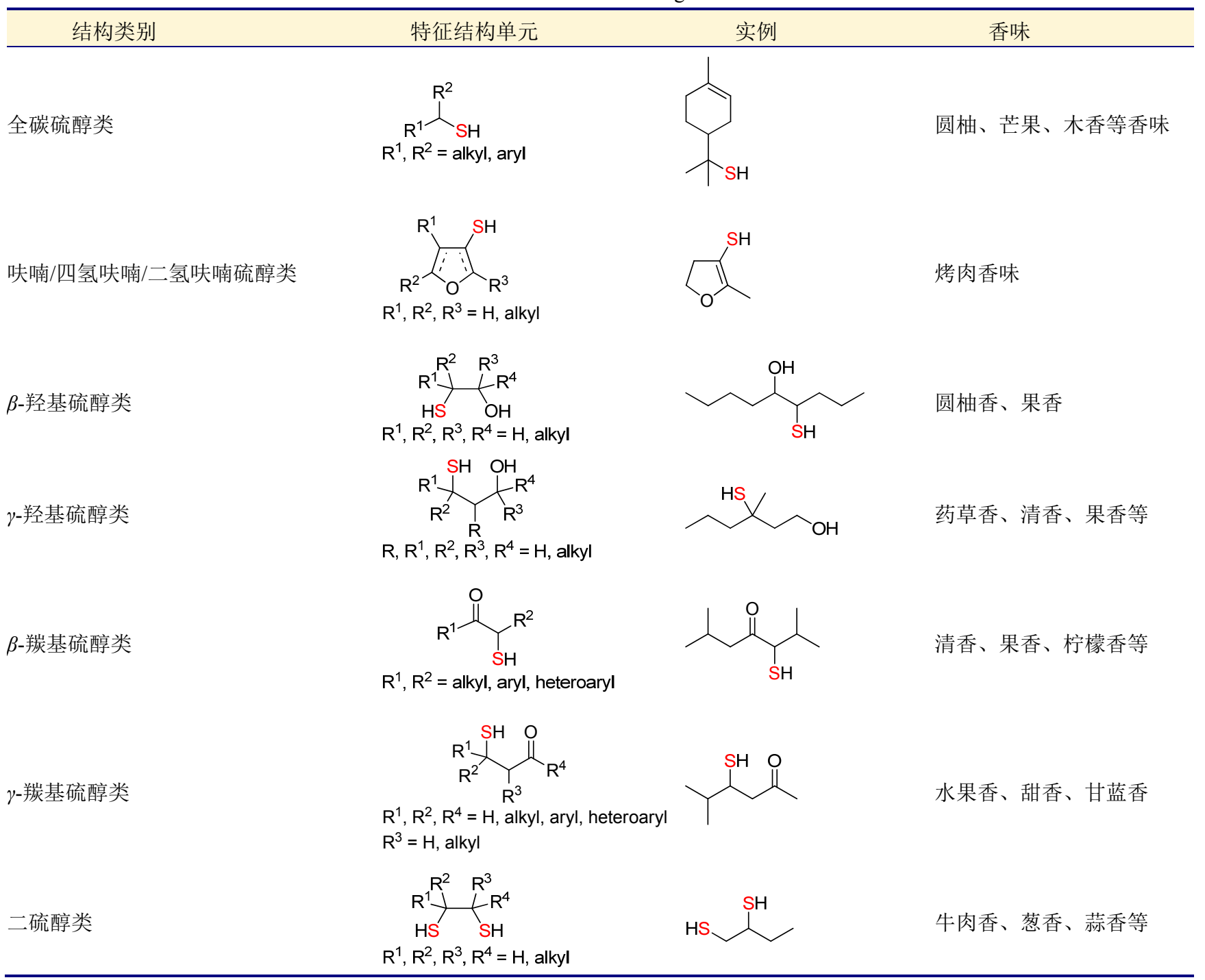

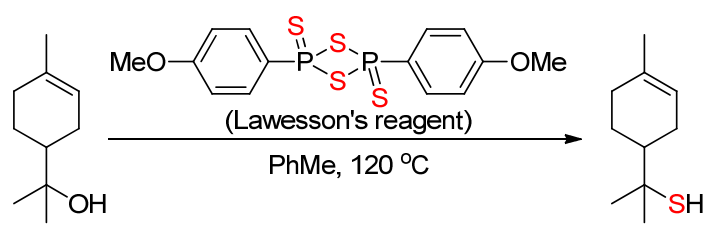

42<smiles>C=CC(C)(O)CCC=C(C)C</smiles>

图式 5 全碳类硫醇的构建

43, $6 \%$ (a)

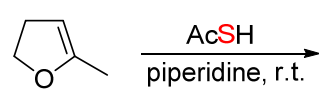

48<smiles>CC1OCCC1[Se]</smiles>

49

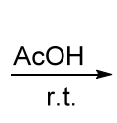

$\underset{\text { r.t. }}{\stackrel{\mathrm{OH}}{\longrightarrow}}$<smiles>CC1OCCC1S</smiles>

$50,87 \%$

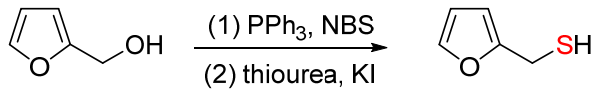

$$
\begin{aligned}
& 51 \\
& \text { 52, 35\% }
\end{aligned}
$$

图式 6 呋喃/四氢呋喃/二氢呋喃类硫醇的构建

Scheme 6 Construction of furan/tetrahydrofuran/dihydrofuran thiol derivatives

(b)

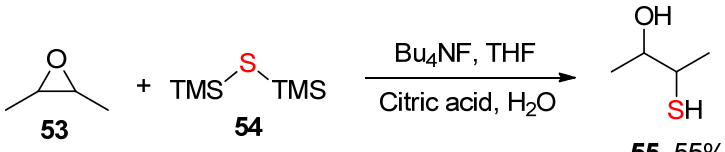

Scheme 5 Construction of all-carbon thiol derivatives 


$$
\begin{aligned}
& \overbrace{\mathrm{OH}}^{\stackrel{\text { Dess-Martin }}{\longrightarrow}} \overbrace{\mathrm{R}} \stackrel{\mathrm{CHO}}{\stackrel{\mathrm{AcSH}}{\text { piperidine }}} \\
& 56 \\
& \mathrm{R}=\mathrm{H} \text {, alkyl } \quad 70 \% \sim 82 \%
\end{aligned}
$$

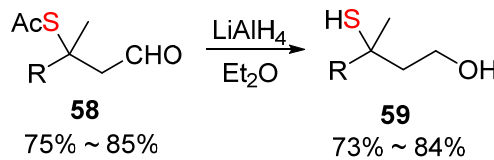

图式 $7 \gamma$-差基硫醇的构建

Scheme 7 Construction of $\beta$-hydroxyl thiol derivatives

进攻得到化合物 62, 最后水解脱去乙酰基即可合成 $\beta$ 羰基硫醇化合物 $\mathbf{6 3}^{[27]}$.<smiles>CCC(=O)C(O)CC</smiles>

60

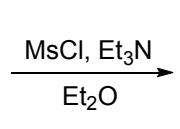

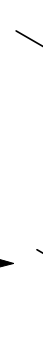

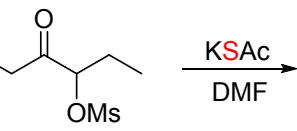

61<smiles>CCC(=O)C(S)CC</smiles>

$63,59 \%$
图式 $8 \beta$-羰基硫醇的构建

Scheme 8 Construction of $\beta$-carbonyl thiol derivatives

$\gamma$-羰基硫醇类化合物很多从植物中提取分离得到, 他们一般表现出水果香、蔬菜香和青香等香气. 例如 $\gamma$ 羰基硫醇类化合物 65 就是从红甜椒中提取分离得到的 香料分子, 具有绿植、蔬菜等香味, 能用作蔬菜香料. 它 可以通过三苯基硅基硫醇对 $\alpha, \beta$-不饱和酮 64 的加成而 制备, 通常的硫化物除了硫醇之外，还可以是硫化氢 (Eq. 4) ${ }^{[28]}$.<smiles>CCC/C=C/C(C)=O</smiles>

64
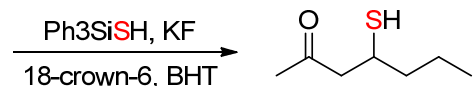

$65,14 \%$
二硫醇类香精香料分子包括邻二硫醇和间二硫醇, 它们具有典型的硫化物香味特点, 主要表现为蒜香、咖 啡香、肉香等, 常用于烤肉、咖啡等多种食用香精香料. 以邻二硫醇的合成为例, 环氧丙烷衍生物 66 与硫氰酸 钾反应得到硫化产物 67 , 再与硫氢化钾开环即可得到 邻二硫醇化合物 68 (Scheme 9) ${ }^{[29]}$.

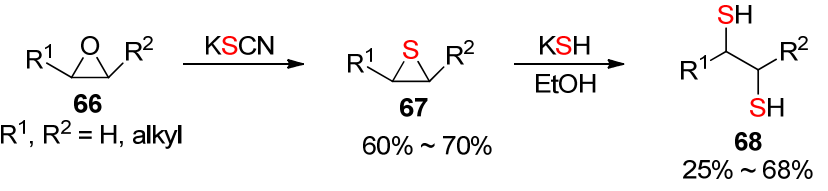

图式 9 二硫醇的构建

Scheme 9 Construction of dithiol derivatives

\section{3 硫酯类}

硫脂类香精香料的结构相对比较单一，主要分为 (杂)芳基-烷基硫脂和烷基一烷基硫脂两大类. 硫脂类香 精香料的合成都是通过相应的硫醇与对应的酰氯缩合 而得.

最具代表性的例子, 如硫代香叶酯 70 具有水果、葱 等的香味，阀值低，尤其具有熏衣草型的香韵特征. 使 得它不仅可以作为食品添加剂，还可以广泛用于牙膏、 香水产品以及芳香制品等. 它的合成是用烷基硫醇 69 与乙酰氯的缩合制备而得(Eq. 5) ${ }^{[30]}$.<smiles>CCN(CC)CCCCC(C)=CCSC(C)=O</smiles>

69

$70,56 \%$

除此之外，硫酯类化合物还可以通过羧酸和硫醇在 缩合剂的作用下缩合得到目标硫酯化合物。例如硫醇 71 与色氨酸衍生物可以缩合得到硫酯化合物 72 (Eq. $6)^{[31]}$.
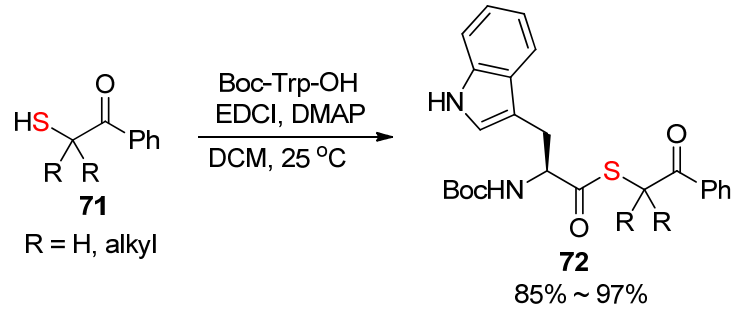

我们小组利用无机硫源的策略可用来调控性高效 构建硫酯和硫醚化合物. 在 Hiyama 反应硫化中, 我们 在钯催化条件下，利用配体调控方式，可以选择性调控 合成硫醚 75 和硫酯 76 两类化合物. 不仅避免了传统使 用恶臭硫醇作为硫源的弊端，还可以同时高效构建两类 含硫化合物(Scheme 10) ${ }^{[32]}$.

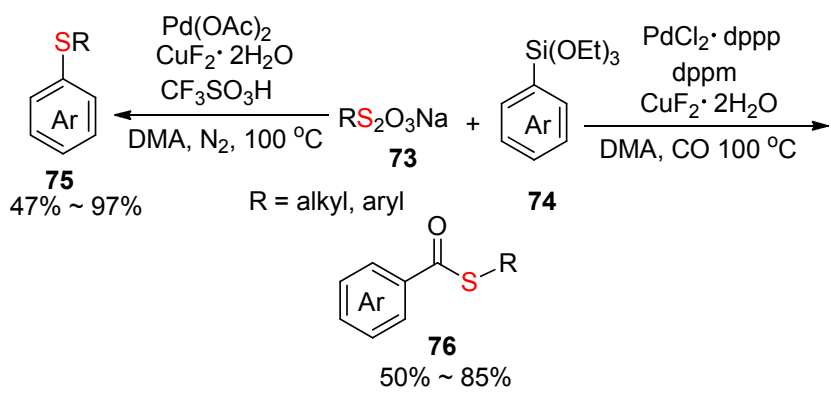

图式 10 硫酯硫醚的选择性构建

Scheme 10 Selective construction of thioester and sulfide derivatives 


\section{4 多硫类}

多硫类化合物广泛存在于香精香料类化合物中, 由 于很多类型的多硫化合物存在于食品的香气成分和水 果中, 因此该类化合物一般具有与食品相关的香气. 例 如蒜香、咖啡香、牛肉香等. 多硫类香精香料根据其含 硫原子的个数, 可以分为如表 3 所示五类化合物.

二硫化合物是多硫香精香料分子中最常见的一类， 二硫的合成以近年发展的无机硫源为例, 对称的烯丙基 过硫化合物 77 可以由烯丙基卤化物与硫脲在 4,4-偶氮 吡啶条件下构建 (Scheme 11a $)^{[33]}$. 环状含三个硫原子化 合物 79 可以用相应的对称烯基过硫化合物 78 为原料,
与硫化氢气体环化得到(Scheme 11b $)^{[34]}$.

三硫化合物常常存在于各种食物中, 例如扇贝和大 蒜中均有三硫化合物. 近年发展的三硫化合物绿色合成 方法包括，直接用无机硫粉作为硫源，在碱的条件下与 烷基卤化物来完成(Scheme 12a $)^{[35]}$. 四硫化合物也是香 精香料工业上使用到的一类化合物，以四硫化合物 83 合成为例, 乙基过硫 81 与氯代三苯基甲基过硫化合物 82 在醋酸条件下可以方便得到四硫化合物 83 (Scheme $12 b)^{[36]}$. 另外, 四硫化合物也可以直接由硫醇与二氯二 硫来构建(Scheme 12c $)^{[37]}$.

表 3 多硫类香精香料分子特征结构和香味

Table 3 The structure of polysulfide-containing flavors and their smell

\begin{tabular}{|c|c|c|c|}
\hline 结构类别 & 特征结构单元 & 实例 & 香味 \\
\hline 二硫类 & $\begin{array}{c}R^{1-S_{-}-R^{2}} \\
R^{1}, R^{2}=\text { alkyl, aryl, heteroaryl }\end{array}$ & & 大蒜等香味 \\
\hline 环状二硫类 & $\begin{array}{l}R^{1} N_{n} R^{2} \\
R^{1}, R^{2}=H \text {, alkyl }\end{array}$ & & 烤肉香味 \\
\hline 三硫类 & $\begin{array}{c}R^{1-S} S^{-S} S^{2} \\
R^{1}, R^{2}=\text { alkyl, aryl, heteroaryl }\end{array}$ & & 肉香、奶香等 \\
\hline 环状三硫类 & $\begin{array}{l}{ }_{\mathrm{R}} \mathrm{R}_{n} \mathrm{H} \text {, alkyl } \\
\mathrm{R}\end{array}$ & & 烤肉香等 \\
\hline 四硫类 & 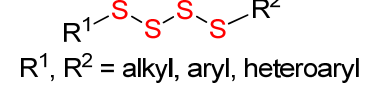 & & 牛肉香等 \\
\hline
\end{tabular}<smiles>C=CC[B]NC(N)=S</smiles><smiles>CC(C)[B]SC(C)C(C)O</smiles>

$80,82 \%$

$77,95 \%$

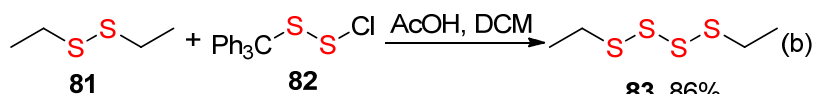<smiles>C=C[Se]SC=CCCCOc1ccccc1</smiles>

(b)

图式 11 二硫类化合物构建 Scheme 11 Construction of disulfides

近年来, 我们小组 ${ }^{[38]}$ 利用无机硫盐发展了一系列 构建非对称过硫化合物的方法. 2015 年, 我们小组报道

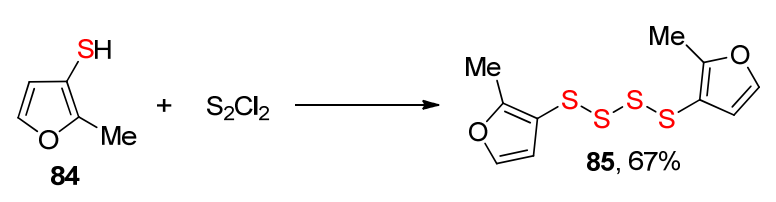

图式 12 多硫类化合物的构建 Scheme 12 Construction of polysulfides 
了以两种廉价易得的无臭 “含硫无机盐” 硫代硫酸钠盐 $\left(\mathrm{R}^{1} \mathrm{~S}_{2} \mathrm{O}_{3} \mathrm{Na}\right)$ 和亚磺酸钠盐 $\left(\mathrm{R}^{2} \mathrm{SO}_{2} \mathrm{Na}, 86\right)$ 为原料, 通过价 态归中策略构建过硫结构 87 (Eq. 7). 硫代硫酸钠盐可 以由烷基卤化物与硫代硫酸钠在反应体系中原位生成.

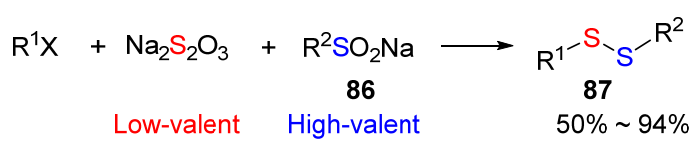

2016 年我们课题组 ${ }^{[39]}$ 又设计了一类新型无臭、稳 定过硫化试剂 90 (Scheme 13a). 该试剂的设计理念基于 之前所提出的 “面具效应” 策略: 引入吸电子官能团乙 酰基, 既可增加弱键 $\mathrm{S}-\mathrm{S}$ 键的稳定性, 还可降低硫对 金属的毒化作用和颈基的恶臭. 运用这一新试剂, 有效 控制醇释放速度, 就可与金属偶联试剂进行 “安装”。与 商业易得的各类嗍酸 91 通过廉价金属铜催化、氧气作 为氧化剂、绿色环保的乙醇作为溶剂、室温条件下，方 便且高兼容性地一步引入两个硫(Scheme 13b). 克级规 模与重要生命分子(氨基酸、糖)都可得到高效过硫化结 构 92.
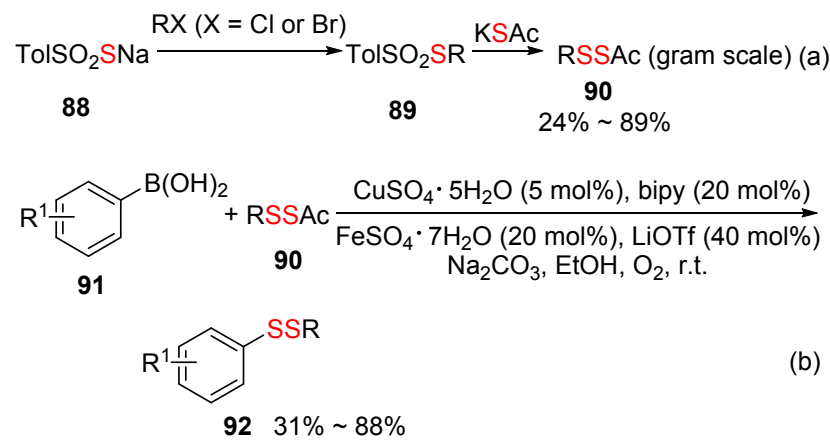

图式 13 氧化偶联构建过硫

Scheme 13 Oxidative cross-couplings reaction for the synthesis of disulfides
2018 年, 我们课题组再次以 “面具效应” 理念，报 道了一类新型亲电过硫试剂(RSSOMe), 实现了与多种 亲核试剂的过硫化反应. 试剂以甲氧基( $\mathrm{OMe}$ )作为面具 基团, 从而使相连的硫原子极性反转 $(\mathrm{RSS}+)$, 并且通 过该面具的电子效应稳定了硫硫键. 在室温条件下，以 廉价金属硫酸铜 $(0.25 \mathrm{~mol} \%)$ 为催化剂, 完成了亲电过硫 化试剂的克级规模建立，为其合成应用奠定了量的基 础. 运用该试剂与碳亲核试剂(富电子芳环、吲哚和吡咯 杂环、1,3-二羰基化合物)、氮亲核试剂(苯胺、磺胺、氨 基酸和来那度胺等)及硫亲核试剂的多样性偶联，建立 了丰富的多硫结构化合物库(Scheme 14) ${ }^{[40]}$.

2018 年，徐政虎小组发展了磺酰基保护的过硫化 合物 97 作为过硫源，与嗍酸和 1,3-二羰基用于构建非对 称的过硫化合物 98 和 99 . 另外, 该过硫源还可以与硫 醇(或硫酚)用来构建三硫化合物 96. 这一系列的亲电性 多硫化反应均可在温和的条件下完成(Scheme 15) ${ }^{[41]}$.

\section{5 结论}

随着国民经济的持续增长，人民生活水平的不断提 高, 含硫香精香料的需求量也在不断增加, 含硫香料的 生产规模也在不断的扩大，其合成方法的研究也在不断 的深入. 然后, 目前的合成方法中大部分仍然需要使用 环境不友好、具有恶臭的硫醇作为基本原料，在强调人 与自然和谐发展的今天，绿色化合成需求量不断增加的 含硫香精香料显得尤为重要. 尤其在含硫香精香料的工 业生产中, 异味、剧毒等环境不友好的化学品已经被禁 止使用，因此，发展绿色无臭无污染的硫化方法将是含 硫香精香料类化合物构建的大势所趋. 同时，含硫香精 香料需求的持续增加也促进了优良的硫化物构建方法 的快速发展.

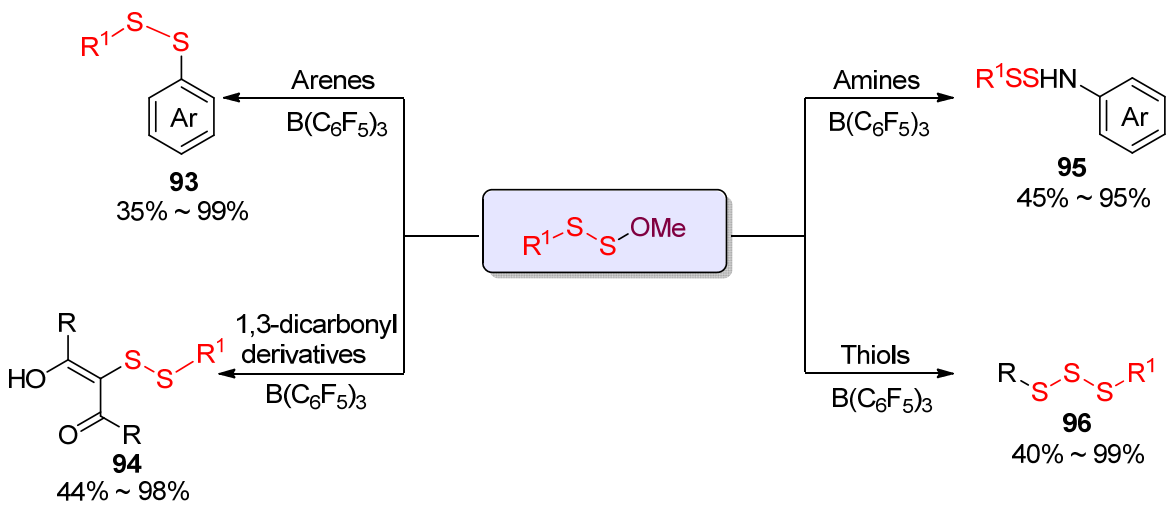

图式 14 亲电多硫化试剂

Scheme 14 Electrophilic polysulfurating reagent 


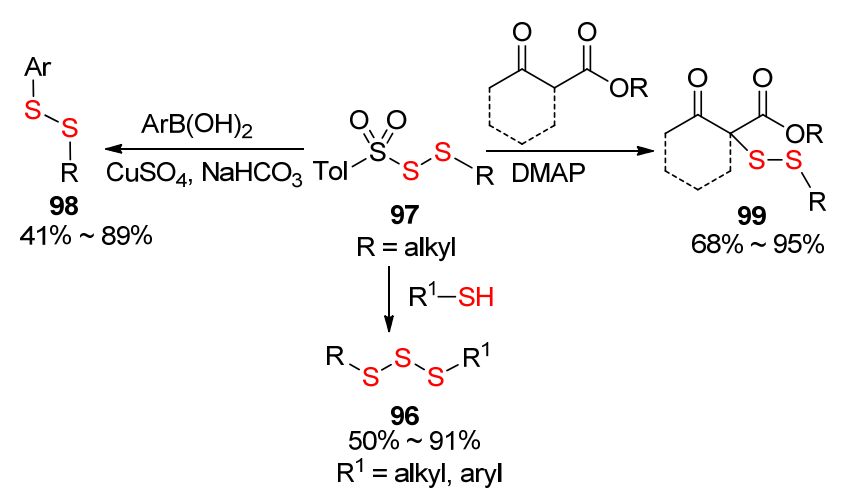

图式 15 氧化偶联构建过硫

Scheme 15 Oxidative cross-coupling reactions for the synthesis of disulfides

\section{References}

[1] Wei, W. Textbook of Organic Chemical Industry, Vol. 3, Chemical Industry Press, Beijing, 1990, p. 206 (in Chinese).

(魏文德, 有机化工原料大全, 第三卷, 化学工业出版社, 北京, 1990, p. 206.)

[2] Smith, R. L.; Doull, J.; Feron, V. J.; Goodman, J. I.; Munro, I. C.; Newberne, P. M.; Portoghese, P. S.; Waddell, W. J.; Wagner, B. M.; Adams, T. B.; Mcgowen, M. M. Food Technol. 2001, 55, 34.

[3] Cohen, S. M.; Fukushima, S.; Gooderham, N. J.; Hecht, S. S.; Marnett, L. J.; Rietjens, I. M. C. M.; Smith, R. L.; Bastaki, M.; McGowen, M. M.; Harman, C.; Taylor, S. V. Food Technol. 2015, $69,40$.

[4] Sun. B. G. Sulfur-Containing Fragrance Chemistry, Science Press, 2007 (in Chinese).

(孙宝国, 含硫香料化学, 科学出版社, 2007.)

[5] Mosciano, G. Perfum. Flavor. 1998, 23, 33.

[6] Tehrani, K. A.; Boeykens, M.; Tyvorskii, V. I.; Kulinkovich, O.; Kimpe, N. D. Tetrahedron 2000, 56, 6541.

[7] (a) Seyedi, S. M.; Sadeghian, H.; Safari, Z. Phosphorus, Sulfur Silicon Relat. Elem. 2009, 184, 2297.

(b) Huang, M.; Tian, H.; Sun, B. Fine Chem. 2005, 22, 127 (in Chinese).

(黄明泉，田红玉，孙宝国，精细化工, 2005, 22, 127.)

[8] Wang, Y.-L.; Tian, H.; Sun, B.; Yu, J. China Surfactant Deterg. Cosmet. 2010, 40, 448 (in Chinese).

(王亚玲，田红玉，孙宝国，余洁菲，日用化学工业，2010，40， 448.)

[9] Lin, Y.; Yi, W.; Shen, W.; Lu, G. Org. Lett. 2016, 18, 592.

[10] Lin, Y.; Lu, G.; Wang, G.; Yi, W. Adv. Synth. Catal. 2016, 358, 4100 .

[11] (a) Wang, S. CN 101607948, 2009

(b) Sun, B.; Tian, H.; Huang, M.; Liu, Y.; Xie, J.; Zheng, F. CN 1709867, 2005
[12] Singh, S.; Kumar, S.; Chimni, S. S. Tetrahedron: Asymmetry 2001 $12,2457$.

[13] Guo, A. China Condiment 2009, 10, 96 (in Chinese). (郭安齐, 中国调味品, 2009, 10, 96.)

[14] Brown, H.; Lynch, G. J. J. Org. Chem. 1981, 46, 930.

[15] Burk, M. J.; Gerlach, A.; Semmeril, D. J. Org. Chem. 2000, 65, 8933-8939.

[16] Sakai, N.; Moritaka, K.; Konakahara, T. Eur. J. Org. Chem. 2009 , 4123-4127.

[17] Liu, X.; Long, S.; Mao, Y.; Diao, H.; Kong, Q.; Wang, Y.; Guo, F. CN 102127038, 2011.

[18] Schoenauer, S.; Schieberle, P. J. Agric. Food Chem. 2018, 66, 4189.

[19] (a) Russell, G. A.; Law, W. C.; Zaleta, M. J. Am. Chem. Soc. 1985, 107,4175 .

(b) Mussinan C. J.; Giacino, C.; WaIradt, J. P. DE 2426865, 1975.

[20] Schoenauer, S.; Schieberle, P. J. Agric. Food Chem. 2016, 64, 3849-3861.

[21] (a) He, K.; Xu, F.; Li, Q.; Wang, G.; Zhu, G. CN 108440457, 2018. (b) Bom, D. C.; Christenson, P. A.; Lam, A. M.; Zhang, Y. WO 2012072631, 2011

[22] Stefaniak, M.; Jasiński, M.; Romański, J. Synlett 2015, 26, 1045.

[23] Degl'Innocenti, A.; Capperucci, A.; Cerreti, A.; Pollicino, S.; Scapecchi, S.; Malesci, I.; Castagnoli, G. Synlett 2005, 3063.

[24] Ian, W. J.; Still, L.; Martyn, J. P. Synth. Commun. 1998, 28, 913.

[25] Vermeulen, C.; Christine, G. D.; Collin, S. J. Agric. Food Chem. 2003, 51, 3623.

[26] Polster, J.; Schieberle, P. J. Agric. Food Chem. 2017, 65, 4329.

[27] (a) Tamura, H.; Fujita, A.; Steinhaus, M.; Takahisa, E.; Watanabe, H.; Schieberle, P. J. Agric. Food Chem. 2010, 58, 7368.

(b) Tamura, H.; Fujita, A.; Steinhaus, M.; Takahisa, E.; Watanabe, H.; Schieberle, P. J. Agric. Food Chem. 2011, 59, 10211.

[28] Naef, R.; Velluz, A.; Jaquier, A. J. Agric. Food Chem. 2008, 56, 517.

[29] Wilson, R. A.; Giacino, C. US 3892878, 1975.

[30] Li, Q.; Zheng, L. Hebei Chem. Eng. Ind. 2006, (2), 24 (in Chinese) (李庆奎, 郑连义, 河北化工, 2006, (2), 24).

[31] Hatanaka, T.; Yuki, R.; Saito, R.; Sasaki, K. Org. Biomol. Chem. 2016, 14,10589

[32] Qiao, Z.; Jiang, X. Org. Lett. 2016, 18, 1550

[33] Khalili, D.; Iranpoor, N.; Firouzabadi, H. J. Sulfur Chem. 2015, 36, 544.

[34] Zhang, L.; Xiao, Y.; Liu, Y. CN 101824026, 2010

[35] Soleiman-Beigi, M.; Yavari, I.; Sadeghizadeh, F. Phosphorus, Sulfur, Silicon Relat. Elem. 2018, 193, 41.

[36] Rys, A. Z.; Harpp, D. N. Tetrahedron Lett. 2000, 41, 7169.

[37] Evers, W. J. US 4020175, 1977.

[38] Xiao, X.; Feng, M.; Jiang, X. Chem. Commun. 2015, 51, 4208.

[39] Xiao, X.; Feng, M.; Jiang, X. Angew. Chem., Int. Ed. 2016, 55, 14121

[40] Xiao, X.; Xue, J.; Jiang, X. Nat. Commun. 2018, 9, 2191.

[41] Wang, W.; Lin, Y.; Ma, Y.; Tung, C.-H.; Xu, Z. Org. Lett. 2018, 20 , 3829 . 